\title{
Prevalência e complicações das lesões dentárias traumáticas
}

\author{
Prevalence and consequences of traumatic dental injuries
}

\author{
Érica dos Santos Carvalho' ${ }^{1}$ Raiane Adorno Trindade², Bruno Magalhães Morais Silva², Ludmilla Macedo \\ Bacelar², Maria Cristina Teixeira Cangussu ${ }^{3}$, Joana Dourado Martins Cerqueira ${ }^{4 *}$
}

\begin{abstract}
${ }^{1}$ Doutora em Odontologia, Professora Adjunto da Faculdade de Odontologia da Universidade Federal da Bahia (UFBA); ${ }^{2}$ Cirurgião-dentista pela UFBA; ${ }^{3}$ Doutora em Saúde Pública, Professora Associada da Faculdade de Odontologia da UFBA; ${ }^{4}$ Mestre em Saúde Coletiva, Professora do Curso de Odontologia da Unidade de Ensino Superior de Feira de Santana e do Centro Universitário UniFTc.
\end{abstract}

\begin{abstract}
Resumo
Introdução: os traumatismos dentários precisam ser corretamente diagnosticados e tratados desde o primeiro atendimento, com a finalidade de minimizar as alterações tardias que poderão complicar o tratamento endodôntico. Objetivo: avaliar a prevalência dos traumatismos dentários na dentição permanente e apresentar as principais alterações subsequentes a estes. Metodologia: foi realizado um estudo observacional da prevalência de traumatismo dentário na dentição permanente e da ocorrência de possíveis complicações endodônticas pós-traumáticas, por meio do acompanhamento clínico e radiográfico dos pacientes atendidos na disciplina de Endodontia Clínica da Faculdade de Odontologia da Universidade Federal da Bahia, no período de 2012 a 2016. Resultados: os dados obtidos foram tabulados e a análise dos resultados incluiu distribuição de frequência e teste Qui-quadrado com nível de significância de $5 \%$. A amostra compreendeu 178 dentes de 89 pacientes, sendo a sua maioria do gênero masculino (54\%). A faixa etária mais prevalente foi dos 11 aos 20 anos de idade. A maxila foi a região mais afetada, sendo os dentes anteriores os mais acometidos (83\%). A queda foi o fator etiológico predominante (61\%) e a fratura coronária não complicada foi o tipo de trauma mais prevalente $(23,2 \%)$, seguido da luxação lateral $(19,7 \%)$ e da avulsão $(17,7 \%)$. A necrose da polpa foi a complicação endodôntica mais frequente $(42,7 \%)$, seguida da reabsorção radicular externa com 18,3\%. Conclusão: pôde-se observar uma alta ocorrência das complicações pós-traumáticas, sendo necessário o tratamento e o acompanhamento criterioso desses pacientes com o intuito de evitar a perda dentária.
\end{abstract}

Palavras-chave: Endodontia. Epidemiologia. Traumatismos Dentários.

\begin{abstract}
Introduction: dental trauma needs to be correctly diagnosed and treated from the first visit, in order to minimize late changes that may complicate endodontic treatment. Objective: To evaluate the prevalence of dental trauma in permanent dentition and to present the main subsequent changes. Metodology: an observational study of the prevalence of dental trauma in permanent dentition and the occurrence of possible post-traumatic endodontic complications was carried out through clinical and radiographic follow-up of patients treated at the Clinical Endodontics discipline of the Federal University School of Dentistry. Bahia, from 2012 to 2016. Results: the data obtained were tabulated and the analysis of the results included frequency distribution and Chi-square test with a significance level of 5\%. The sample comprised 178 teeth from 89 patients, most of them male (54\%). The most prevalent age group was from 11 to 20 years old. The maxilla was the most affected region, with the anterior teeth being the most affected (83\%). Fall was the predominant etiological factor (61\%) and uncomplicated coronary fracture was the most prevalent type of trauma (23.2\%), followed by lateral dislocation (19.7\%) and avulsion (17.7\%). . Pulp necrosis was the most common endodontic complication (42.7\%), followed by external root resorption with $18.3 \%$. Conclusion: it was observed a high occurrence of post-traumatic complications, requiring the treatment and careful monitoring of these patients in order to prevent tooth loss.

Keywords: Endodontics. Epidemiology. Tooth injuries
\end{abstract}

\section{INTRODUÇÃO}

O traumatismo dentário tem sido considerado um problema de saúde pública, afetando cerca de 4 a $33 \%$ da população, principalmente crianças e adolescentes ${ }^{1-3}$. O aumento da violência na sociedade atual, o grande número de acidentes de trânsito e os acidentes ocasionais em atividades esportivas também têm contribuído para

Correspondente/Corresponding: *Joana Dourado Martins Cerqueira End: Rua Quintas do Sol, $n^{\circ} 20$ Condomínio Quintas do Sol Ville 2, casa 14 Bairro Parque Ipê, Feira de Santana, Bahia - Tel: (55) (75) 3161-8248 -E-mail:martinsjoana_1@hotmail.com transformar a injúria dentária traumática em um problema de saúde em progressão ${ }^{4}$.

O conhecimento sobre essas injúrias e suas sequelas é de interesse dos profissionais de saúde, porque interferem diretamente de uma forma negativa na qualidade de vida dos pacientes ${ }^{5}$. Estudos revelam que as injúrias dentárias são a principal causa de procura das unidades de pronto-atendimento. No entanto, grande parte destes elementos é perdida ou possui uma sobrevida diminuída, devido à demora no atendimento ou à desinformação dos profissionais de saúde a respeito dos planos de tratamentos e proservação destes dentes traumatizados ${ }^{6}$. 
Os traumatismos dentários podem englobar desde as injúrias simples, como trincas ou fraturas de esmalte, até os traumas mais complicados envolvendo polpa e/ ou ligamento periodontal, como em fraturas radiculares, luxações e avulsões. Na ocorrência de lesões complicadas, o tratamento e o prognóstico se tornam dificultados, uma vez que o grau de complexidade é maior, exigindo uma equipe multidisciplinar ${ }^{7,8}$.

As alterações traumáticas podem ser imediatas, necessitando de tratamento no momento da urgência, ou podem surgir de forma mediata, posteriores à lesões na polpa ou periodonto, como necrose pulpar, reabsorção dentária, anquilose, calcificação pulpar e escurecimento coronário ${ }^{9}$. Essas complicações podem ocorrer semanas, meses, ou até mesmo anos depois do traumatismo, portanto, necessitam de um acompanhamento a longo prazo, já que interferem no prognóstico do dente envolvido ${ }^{7,10}$.

A necrose pulpar é a complicação pós-traumática mais comum, principalmente nos casos de fraturas coronárias e radiculares, porém, alguns fatores podem interferir no aparecimento de alterações tardias incluindo o tipo da injúria, o estágio do desenvolvimento radicular, o tempo e tipo de atendimento inicial 7,11. Estudos mostram que existe uma forte relação entre o surgimento das reabsorções radiculares externas e traumatismos mais severos aos tecidos de suporte como luxações e avulsões ${ }^{7,10,12,13}$.

A incidência dessas complicações pós-traumáticas precisa ser minimizada, uma vez que, a presença destas dificultam ou impossibilitam a realização de tratamentos endodônticos, o que pode acarretar na perda progressiva dos dentes permanentes, gerando um importante problema de saúde pública ${ }^{10}$. Diante do exposto, torna-se importante realizar trabalhos que avaliem a prevalência dos traumatismos dentários e a ocorrência de complicações endodônticas que podem surgir nesses dentes traumatizados, com o intuito de melhor prevenir tais sequelas indesejáveis nessa parcela da população.

Dessa forma, a finalidade do presente estudo foi avaliar a frequência dos diferentes tipos de trauma e o padrão de aparecimento das alterações tardias subsequentes, bem como definir as características desta população envolvida, por meio de dados clínicos e radiográficos obtidos nos prontuários de pacientes atendidos no ambulatório de Endodontia da Faculdade de Odontologia da Universidade Federal da Bahia (FOUFBA) no período de 2012 a 2016.

\section{METODOLOGIA}

Trata-se de um estudo retrospectivo que se baseou em uma amostra de conveniência constituída por 89 pacientes, totalizando 178 dentes que sofreram algum tipo de trauma dental e foram atendidos e acompanhados pelo Grupo de Pesquisa em Traumatismos Dentários da disciplina de Endodontia Clínica FOUFBA, no período de 2012 a 2016. Esta pesquisa foi aprovada pelo Comitê de Ética e Pesquisa da mesma faculdade sob o parecer $n$ ㅇ 1.895.087. Todos os pacientes ou seus responsáveis legais foram informados sobre os protocolos de procedimentos, riscos e benefícios em relação à participação e assinaram um termo de consentimento livre e esclarecido autorizando a realização desta pesquisa.

Pacientes com traumatismos em dentes decíduos foram excluídos do estudo, portanto, apenas os dentes permanentes traumatizados de crianças e adultos foram avaliados nesta pesquisa. Foram excluídos também, os prontuários de pacientes que não compareceram periodicamente para o acompanhamento.

Foram analisadas as seguintes informações inerentes ao tipo e número de dentes avaliados, gênero e idade do paciente no momento do trauma, tipo e causa do trauma e ocorrência das complicações. Os tipos de traumatismos dentários foram classificados de acordo com o critério proposto por Andreasen, J.O, Andreasen, F.M e Andersson ${ }^{8 .}$

Um plano de tratamento foi elaborado para cada paciente e todos foram acompanhados periodicamente através da análise clínica e radiográfica. A partir destas visitas periódicas foram registradas as sequelas pós- traumáticas, incluindo a necrose pulpar, calcificação pulpar, reabsorção interna, reabsorção radicular externa e alteração da coroa dentária. Os testes de sensibilidade pulpar foram realizados com um spray de resfriamento à $-50^{\circ} \mathrm{C}$ (Endolce- Maquira, São Paulo-Brasil). As calcificações e as reabsorções foram investigadas e comparadas com as radiografias periapicais convencionais anteriores.

Os dados coletados foram organizados numa planilha Excel (Microsoft, Seattle, WA, USA) e tabulados com a distribuição percentual de ocorrências para cada tópico avaliado. Para a correlação dos tipos de trauma com a alteração tardia e causa do trauma com o gênero, foram realizadas análises estatísticas com o teste Qui-quadrado de Pearson, ao nível de significância de 5\%.

\section{RESULTADOS}

Foram avaliados 89 pacientes com 178 dentes traumatizados. Destes, 48 eram do gênero masculino (54\%) e 41 pacientes do gênero feminino (46\%). A faixa etária mais prevalente foi entre 11 a 20 anos, com 32 pacientes (36\%) e os dentes anteriores superiores (incisivos e caninos) foram os mais acometidos por essas injúrias traumáticas (83\%). A queda da própria altura prevaleceu como sendo o fator etiológico mais comum dos traumas dentais, presente em 54 casos (61\%), seguida das agressões físicas (15\%), práticas esportivas (10\%), acidentes automobilísticos (11\%) e atropelamento (3\%) (Tabela 1).

Dentre os dentes traumatizados, 25 sofreram mais de um tipo de injúria e a frequência do tipo de traumatismo dentário foi em ordem decrescente: Fratura Coronária Não complicada (23,2\%); Luxação lateral (19,7\%); Avulsão $(17,7 \%)$; Subluxação (10,3\%); Fratura Coronária Complicada (7,4\%); Luxação Extrusiva (6,9\%); Luxação Intrusiva $(6,4 \%)$; Fratura Radicular (5,4\%); Fratura Coronorradicular (2\%) e Concussão (1\%) (Gráfico 1).

Pôde-se notar que $22,1 \%$ dos dentes não apresentaram complicações traumáticas tardias, porém, a sequela 
mais prevalente foi a Necrose Pulpar (42,7\%), seguida pela Reabsorção Radicular Externa (18,3\%). Outras alterações foram notificadas com menor frequência como Descoloração da coroa (8,9\%), Calcificação pulpar $(5,6 \%)$ e Reabsorção interna (2,4\%) (Gráfico 2).

A tabela 2 demonstra associação dos fatores etiológicos dos traumatismos com o gênero, podendo-se observar que o gênero masculino foi mais prevalente em todas as causas encontradas, exceto nos acidentes automobilísticos cujo gênero mais acometido foi o feminino.

Tabela 1- Distribuição das variáveis do estudo considerando os números absolutos ( $n$ ) e porcentagens (\%).

\begin{tabular}{lcc}
\hline \multicolumn{1}{c}{ VARIÁVEIS } & $\begin{array}{c}\text { Número } \\
\text { absoluto }(\mathbf{n})\end{array}$ & $\begin{array}{c}\text { Porcentagem } \\
\text { (\%) }\end{array}$ \\
\hline Gênero & & \\
Masculino & 48 & $54 \%$ \\
Feminino & 41 & $46 \%$ \\
Etiologia & & \\
Queda & 54 & $61 \%$ \\
Agressão Física & 13 & $15 \%$ \\
Esportes & 9 & $10 \%$ \\
Acidentes Automobilísticos & 10 & $11 \%$ \\
Atropelamento & 3 & $3 \%$ \\
Faixa etária & & \\
0-10 anos & 9 & $10 \%$ \\
11-20 anos & 32 & $36 \%$ \\
21-30 anos & 17 & $19 \%$ \\
$31-40$ anos & 13 & $15 \%$ \\
$41-50$ anos & 13 & $15 \%$ \\
$51-60$ anos & 5 & $5 \%$ \\
Dentes envolvidos & & \\
Incisivos/Caninos Superiores & 148 & $83 \%$ \\
Incisivos/ Caninos Inferiores & 23 & $13 \%$ \\
Pré-molares & 5 & $3 \%$ \\
Molares & 2 & $1 \%$ \\
\hline
\end{tabular}

Fonte: Grupo de Pesquisa em Traumatismos Dentários da disciplina de Endodontia Clínica FOUFBA

Gráfico 1 - Distribuição do tipo de traumatismo dentário encontrado no estudo considerando os seus valores percentuais (\%).

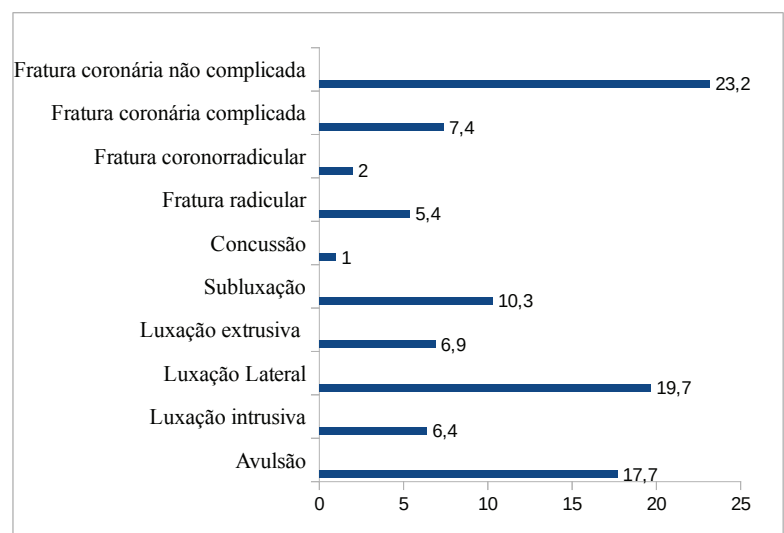

Fonte: Grupo de Pesquisa em Traumatismos Dentários da disciplina de Endodontia Clínica FOUFBA
Gráfico 2- Distribuição das alterações traumáticas tardias encontradas no estudo considerando os seus valores percentuais (\%).

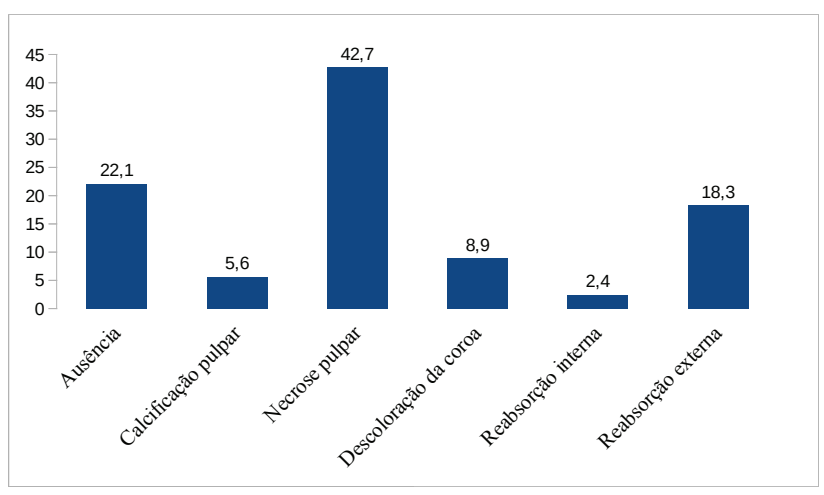

Fonte: Grupo de Pesquisa em Traumatismos Dentários da disciplina de Endodontia Clínica FOUFBA

Tabela 2 - Análise da correlação dos fatores etiológicos do traumatismo dentários e o gênero.

\begin{tabular}{lrrr}
\hline \multicolumn{1}{c}{$\begin{array}{c}\text { ETIOLOGIA } \\
\text { DO TRAUMA }\end{array}$} & \multicolumn{3}{c}{ GÊNERO } \\
\hline Queda & Feminino & Masculino & \multicolumn{1}{c}{ Total } \\
Agressão física & $27(30,3 \%)$ & $30(33,7 \%)$ & $57(64 \%)$ \\
Esporte & $4(4,5 \%)$ & $7(7,9 \%)$ & $11(12,4 \%)$ \\
Acidentes automobilístico & $1(1,1 \%)$ & $7(7,9 \%)$ & $8(9 \%)$ \\
Atropelamento & $8(9 \%)$ & $2(2,2 \%)$ & $10(11,2 \%)$ \\
Total & $0(0 \%)$ & $3(3,4 \%)$ & $3(3,4 \%)$ \\
\hline
\end{tabular}

Pearson Chi-Square P-Value $=0,023$ -

Fonte: Grupo de Pesquisa em Traumatismos Dentários da disciplina de Endodontia Clínica FOUFBA

Na tabela 3, ao correlacionar o tipo de traumatismo dentário com a alteração tardia encontrada, observou-se que a Necrose pulpar foi a alteração mais frequente, exceto nos traumas de Concussão e Subluxação. A reabsorção interna apresentou baixa prevalência e só ocorreu nas fraturas coronárias não complicadas $(1,2 \%)$ e na subluxação $(0,6 \%)$. Nos casos de avulsão dentária, as reabsorções radiculares externas (6\%) e a necrose pulpar (6\%) foram as alterações tardias mais prevalentes, com diferenças estatisticamente significantes. 
Tabela 3 - Análise da correlação entre o tipo de trauma e as alterações dentárias que ocorrem tardiamente.

\begin{tabular}{|c|c|c|c|c|c|c|c|}
\hline Tipo de Trauma & & Alteração & Tardia & & & & \\
\hline & Ausência & $\begin{array}{l}\text { Calcificação } \\
\text { pulpar }\end{array}$ & $\begin{array}{l}\text { Descoloração } \\
\text { coronária }\end{array}$ & $\begin{array}{l}\text { Necrose } \\
\text { pulpar }\end{array}$ & $\begin{array}{l}\text { Reabsorção } \\
\text { interna }\end{array}$ & $\begin{array}{c}\text { Reabsorção } \\
\text { Externa }\end{array}$ & Total \\
\hline $\begin{array}{l}\text { Fratura coronária não com- } \\
\text { plicada }\end{array}$ & $12(7,3 \%)$ & $1(0,6 \%)$ & $5(3 \%)$ & $17(10,3 \%)$ & $2(1,2 \%)$ & $5(3 \%)$ & $42(25,4 \%)$ \\
\hline $\begin{array}{l}\text { Fratura coronária compli- } \\
\text { cada }\end{array}$ & $4(2,4 \%)$ & $0(0 \%)$ & $1(0,6 \%)$ & $10(6 \%)$ & $0(0 \%)$ & $0(0 \%)$ & $15(9 \%)$ \\
\hline Fratura coronorradicular & $1(0,6 \%)$ & $0(0 \%)$ & $0(0 \%)$ & $2(1,2 \%)$ & $0(0 \%)$ & $0(0 \%)$ & $3(1,8 \%)$ \\
\hline Fratura radicular & $0(0 \%)$ & $4(2,4 \%)$ & $0(0 \%)$ & $3(1,8 \%)$ & $0(0 \%)$ & $2(1,2 \%)$ & $9(5,5 \%)$ \\
\hline Concussão & $1(0,6 \%)$ & $1(0,6 \%)$ & $0(0 \%)$ & $0(0 \%)$ & $0(0 \%)$ & $0(0 \%)$ & $2(1,2 \%)$ \\
\hline Subluxação & $17(10,3)$ & $2(1,2 \%)$ & $0(0 \%)$ & $1(0,6 \%)$ & $1(0,6 \%)$ & $0(0 \%)$ & $21(12,7 \%)$ \\
\hline Luxação extrusiva & $0(0 \%)$ & $0(0 \%)$ & $0(0 \%)$ & $11(6,7 \%)$ & $0(0 \%)$ & $1(0,6 \%)$ & $12(7,3 \%)$ \\
\hline Luxação lateral & $9(5,5 \%)$ & $1(0,6 \%)$ & $0(0 \%)$ & $21(12,7 \%)$ & $0(0 \%)$ & $1(0,6 \%)$ & $32(19,4 \%)$ \\
\hline Luxação intrusiva & $0(0 \%)$ & $1(0,6 \%)$ & $2(1,2 \%)$ & $2(1,2 \%)$ & $0(0 \%)$ & $0(0 \%)$ & $5(3 \%)$ \\
\hline Avulsão & $3(1,8 \%)$ & $1(0,6 \%)$ & $0(0 \%)$ & $10(6 \%)$ & $0(0 \%)$ & $10(6 \%)$ & $24(14,7 \%)$ \\
\hline TOTAL & $47(28,5 \%)$ & $11(6,7 \%)$ & $8(4,8 \%)$ & $77(46,7 \%)$ & $3(1,8 \%)$ & $19(11,5 \%)$ & $165(100 \%$ \\
\hline
\end{tabular}

Pearson Chi-Square $(p<0,000)$.

Fonte: Grupo de Pesquisa em Traumatismos Dentários da disciplina de Endodontia Clínica FOUFBA

Figura 1 - a)Imagem clínica de trauma dentário com fratura coronária não complicada e a imagem radiográfica do caso em b) mostrando reabsorção radicular interna e externa. Em c) imagem clínica de Subluxação e em d) a imagem radiográfica do caso mostrando fratura radicular.
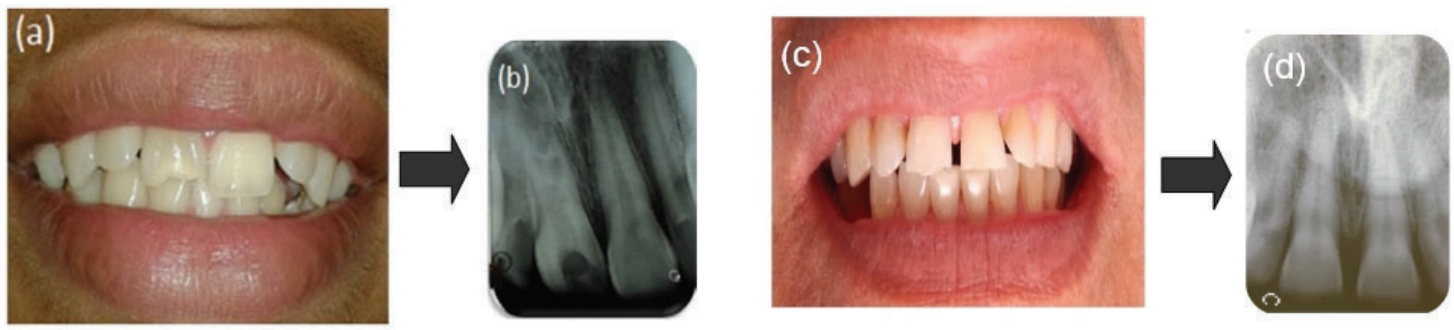

Fonte: Grupo de Pesquisa em Traumatismos Dentários da disciplina de Endodontia Clínica FOUFBA

\section{DISCUSSÃO}

A partir dos dados obtidos nesse estudo foi possível observar que dentre os 89 pacientes atendidos, houveram 178 dentes traumatizados. Destes pacientes avaliados, $53 \%$ pertenciam ao gênero masculino, e conforme literatura, os homens são mais enérgicos e inclinados para atividades ao ar livre, possuindo uma vida social com trabalho e esporte mais perigosos, estando mais propensos os traumatismos dentários ${ }^{14,15-18}$. Entretanto, taxas mais elevadas de ocorrência feminina nos casos de traumatismos dentários, podem ocorrer pelo aumento da participação das mulheres nos esportes ou atividades físicas de impacto anteriormente praticadas por homens ou nos acidentes automobilísticos que não tem predileção por gênero ${ }^{19}$.

Alguns estudos afirmam que a faixa etária mais acometida pelo traumatismo dentário é a primeira década de vida, uma vez que crianças e jovens possuem adversidades físicas e culturais, estando mais envolvidos em atividades de esporte e lazer ${ }^{10,15,17}$. Neste levantamento, a faixa etária mais acometida foi a segunda década de vida (11 a 20 anos), provavelmente por se tratar de uma avaliação em que os dentes decíduos traumatizados foram excluídos e o fator etiológico mais prevalente ter sido a queda, que pode acontecer em qualquer faixa etária ${ }^{10,18}$. Ainda com relação ao fator etiológico do traumatismo, vale ressaltar que a agressão física surgiu em segundo lugar, o que nos alerta para cuidados com as relações sociais e o aumento da violência em nossa sociedade.

Os incisivos e caninos superiores foram os dentes mais afetados, sendo este dado unânime na literatura ${ }^{14-19}$. Isto pode ser explicado pela maior sobressaliência da arcada superior que recebe primeiramente o impacto na região bucal, aumentando a predisposição à injúria dentária ${ }^{16,20}$.

As fraturas coronárias envolvendo esmalte e ou dentina sem exposição pulpar (não complicadas) foram as injúrias traumáticas mais prevalentes, dado que está 
de acordo com outros estudos 10,15,16,20,21. Em seguida, a luxação lateral e a avulsão também apresentaram uma prevalência significante, correspondendo aos traumatismos aos tecidos de sustentação mais comuns $\mathbf{s}^{5,21,22}$.

O surgimento das alterações pós-traumáticas depende principalmente do tempo e do tipo de atendimento inicial, contudo, a necrose pulpar foi a alteração tardia mais observada, ocorrendo em todos os tipos de traumatismos, exceto na concussão ${ }^{8}$. Essa ocorrência se deve ao fato de que dentes com exposição de túbulos de dentina sem a adequada restauração imediata, ou com a polpa exposta à cavidade bucal estão sujeitos à invasão bacteriana e posterior necrose pulpar. Fato semelhante ocorre nos casos de deslocamentos devido às luxações ou às avulsões, onde ocorre o rompimento do feixe vásculo-nervoso apical aumenta essa possibilidade de necrose ${ }^{5,7}$. A reabsorção inflamatória interna, por outro lado, requer vitalidade pulpar para ativar os odontoclastos, sendo então um achado pouco frequente nos dentes traumatizados neste estudo $(2,4 \%)$ e em outros também ${ }^{10,23}$.

As reabsorções radiculares externas também foram alterações tardias frequentes e surgiram principalmente nos casos de luxações severas e avulsões, pois causam danos irreversíveis à camada cementoblástica, desencadeando no processo reabsortivo na superfície radicular externa ${ }^{13,24}$. Nos casos de fraturas coronárias, as reabsorções radiculares externas apenas surgiram no terço apical, devido à associação com lesões periapicais provenientes de infecções endodônticas.

O processo de reabsorção radicular inflamatória pode se instalar em torno de duas semanas após o trauma e atua de forma rápida e progressiva ${ }^{24}$. Já a reabsorção substitutiva, ocorre com a união entre osso alveolar e estrutura radicular, sendo a substância radicular gradualmente substituída por osso ${ }^{13}$. Assim, o atraso na procura de tratamento causa um atraso na extração da polpa, que pode interferir com a progressão de reabsorção radicular, da mesma forma que a não realização de uma contenção adequada pode interferir no prognóstico periodontal.

As calcificações pulpares $(5,6 \%)$ e o escurecimento coronário $(8,9 \%)$ foram as alterações tardias menos frequentes e representam uma resposta degenerativa da polpa, onde o controle do fluxo sanguíneo para os odontoblastos encontra-se alterado, resultando em produção descontrolada de dentina reparadora que pode afetar a translucidez do dente e resultar na opacificação gradual da coroa ${ }^{23,25}$. Essa complicação pós-traumática pode ser observada cerca de um ano após o trauma, entretanto, muitos pacientes envolvidos nesse estudo tiveram um período de acompanhamento inferior, justificando a baixa prevalência dessa alteração com relação a outros estudos ${ }^{10,23,26}$. Além disso, alguns autores têm relatado que o risco de calcificação pulpar é maior em dentes permanentes com rizogênese incompleta, que também não foram muito frequentes nesta amostra ${ }^{25}$.

A subluxação é uma lesão caracterizada por danos ao ligamento periodontal causando mobilidade e um leve deslocamento. O presente estudo demonstrou que a maioria das ocorrências de subluxação não teve complicação pós-trauma e que houve recuperação da polpa e do ligamento periodontal, visto também em outros estudos ${ }^{10,27}$. Contudo, nos casos onde se associa este trauma à uma lesão concomitante, como a fratura de coroa, os mecanismos de defesa da polpa tornam-se menos eficientes e a possibilidade de necrose é elevada ${ }^{28}$.

Diante do exposto, pode-se inferir que as injúrias traumáticas requerem tratamento de urgência rápido e eficiente, porém a cessação dos sinais e sintomas, não significa a cura definitiva do dente traumatizado. Esses dentes precisam ser acompanhados periodicamente por um longo período, prevenindo ou tentando interromper as alterações tardias supostamente possíveis, as quais podem acarretar comprometimento estético, fonético ou da mastigação. Os dados apresentados refletem a necessidade de os profissionais da saúde estarem aptos a diagnosticar e orientar os pais e/ou responsáveis sobre a melhor conduta a ser adotada diante de um trauma dentário.

\section{CONCLUSÃO}

A partir dos dados obtidos nesse estudo foi possível concluir que a maior parte das injúrias traumáticas ocorreu em pacientes do gênero masculino, com faixa etária entre 11 e 20 anos de idade. Os incisivos superiores foram os dentes mais acometidos e o fator etiológico mais prevalente foi a queda. As fraturas coronárias não complicadas foram o tipo de traumatismo mais frequente, e a necrose pulpar foi a alteração tardia mais prevalente. As complicações traumáticas podem aparecer num período mais curto, como as reabsorções radiculares, ou levarem anos para aparecer como as calcificações. Assim, faz-se necessário a implantação de programas de prevenção e esclarecimento da população sobre a importância do acompanhamento clínico e radiográfico desses pacientes.

\section{REFERÊNCIAS}

1. BAXEVANOS, K. et al. Psychosocial factors and traumatic dental injuries among adolescents. Community Dent Oral Epidemiol., Copenhagen, v. 45 , p. 449-457, 2017.

2. BONFIM, R. A; HERRERA, D. R; DE-CARLI A. D. Oral health-related quality of life and risk factors associated with traumatic dental injuries in Brazilian children: A multilevel approach. Dent. Traumatol., Copenhagen, v. 33, p. 358-368, 2017.

3. DAMÉ-TEIXEIRA, N. et al. Traumatic dental injury among 12-year-old South Brazilian schoolchildren: prevalence, severity and risk indicators. Dent. Traumatol., Copenhagen, v. 29, p.52-58, 2013.

4. RAMOS-JORGE, M. L, et. al. Incidence of dental trauma among adolescents: a prospective cohort study. Dent. Traumatol., Copenhagen, v. 24 , p. $159-163,2008$.

5. CARVALHO, R. G. Estudo epidemiológico de pacientes atendidos no Projeto de Trauma Dental no período de 7 anos. Dissertação [Mestrado] - Universidade do Grande Rio "Prof José de Souza Herdy" Escola de Odontologia, Duque de Caxias, 2007. 
6. PETROVIC, B. et al. Factors related to treatment and outcomes of avulsed teeth. Dent. Traumatol., Copenhagen, v. 26, p. 52-59, 2010.

7. LIN, S. et al. Occurrence and timing of complications following traumatic dental injuries: A retrospective study in a dental trauma department. J. Clin. Exp. Dent., Spain, v. 8, n. 4, p. 429-436, 2016.

8. ANDREASEN, J. O.; ANDREASEN, F. M.; ANDERSSON, L. Textbook and color atlas of traumatic injuries to the teeth. 4 ed. Oxford: Blackwell, 2007.

9. MORELLO, J. et al. After-effects following traumatic dental Injury with endodontic involvement. Rev. Bras. Pesqui. Saúde, Vitória, v. 13, n.2, p. 68-73, 2011.

10. HECOVA, H. et al. A retrospective study of 889 injured permanent teeth. Dent. Traumatol., Copenhagen, v. 26, p. 466-475, 2010.

11. ANDRADE, E. S. et al. Root healing after horizontal fracture: a case report with a 13-year follow up. Dent. Traumatol., Copenhagen, v. 24, p.1-3, 2008.

12. CARVALHO, E. S. et al. Root surface treatment using diode laser in delayed tooth replantation: radiographic and histomorphometric analyses in rats. Dent. Traumatol., Copenhagen, 2012.

13. LIMA, T. F. R. et al. Relationship between Initial Attendance after Dental Trauma and Development of External Inflammatory Root Resorption. Braz. Dent. J., Ribeirão Preto, v. 28, n. 2, p. 201-205, 2017.

14. CARVALHO, R. G. et al. Estudo epidemiológico das fraturas coronárias em pacientes atendidos em um projeto de trauma dental em um período de 6 anos. Rev. Bras. Odontol., Rio de Janeiro, v.70, n.1, p. 4-7, 2013.

15. PEREIRA, A.C. et al. Atendimentos realizados no Serviço de Traumatismos Dentários da FOP- Unicamp durante o período de dois anos. RFO, Passo Fundo, v. 21, n.1, p. 9-14, 2016.

16. PELLI PAIVA, P. C. et al. Prevalence and risk factors associated whith traumatic dental injury among 12-year-old schoolchildren in Montes Claros, Mg, Brazil. Ciênc. Saúde Colet., Rio de Janeiro, v. 4, n. 20, p. 1225-1233, 2015.

17. ABAD, E.C. et al. Frequency of coronary fractures without pulp exposure of a reference service in a 7-year period. Braz. J. Den Traum., [s.I], v. 2, n.1, p.22-26, 2010.

18. PIRAGIBE JÚNIOR, M. et al. Fraturas coronárias com exposição pulpar: levantamento epidemiológico em um período de 8 anos. Rev. Bras. Odontol., Rio de Janeiro, p.180-185, 2012.

19. SULIEMAN, A.G.; AWOODA, E. M. Prevalence of anterior dental trauma and its associated factors among preschool children aged 3-5 years in Khartoum city, Sudan. Int. J. Dent.,, Cairo, v. 2018, 2018.

20. VÉLIZ, A. et al. Estudio retrospectivo de casos atendidos en clínica de traumatología dentoalveolar En Santiago, Chile. Int. J. Odontostomat., Temuco, v. 11, n.4, p. 405-410, 2017.

21. ZOU, J. et al. Common dental diseases in children and malocclusion. Int. J. Oral Sci., China, v.10, n.7, 2018.

22. SOUZA-FILHO, F. J. et al. Avaliação das injúrias dentárias observadas no Centro de Trauma Dental da Faculdade de Odontologia de PiracicabaUnicamp, RFO, Passo Fundo, v. 14, n. 2, p. 111-116, maio/ago. 2009.

23. SOARES, A. J. et al. Frequency of root resorption following trauma to permanent teeth. J. Oral Sci., Tokyo, v.57, n. 2, p.73-78, 2015.

24. SILVEIRA, L. F. M. et al. Frequência de reabsorção radicular inflamatória decorrente de trauma em dentes anteriores. RFO, Passo Fundo, v. 18, n.2, p. 185-192, 2013.

25. BASTOS, J. V.; CÔRTES, M. I. S. Pulp canal obliteration after traumatic injuries in permanent teeth - scientific factor fiction? Braz. Oral Res., São Paulo, v. 32, n. 75, 2018.

26. ABD-ELMEGUID, A.; ELSALHY, M.; YU, D. C. Pulp canal obliteration after replantation of avulsed immature teeth: a systematic review. Dent. Traumatol., Copenhagen, v. 31, n.6, p. 437-441, 2015.

27. LIN, S. et al. Occurrence and timing of complications following traumatic dental injuries: A retrospective study in a dental trauma department. J. Clin. Exp. Dent., Spain, v. 8, n.4, p. 429-436, 2016.

28. ZALECKIENE, V. et al. Traumatic dental injuries: etiology, prevalence and possible outcomes. BDM J., [s.I], v. 16, p. 7-14, 2014.

Submetido em: 13/08/2019

Aceito em: 25/08/2020 